\title{
Do triceps surae muscle dynamics govern non-uniform Achilles tendon deformations?
}

\author{
William H. Clark ${ }^{1}$, Jason R. Franz ${ }^{\text {Corresp. } 1}$ \\ ${ }^{1}$ Joint Department of Biomedical Engineering, University of North Carolina at Chapel Hill and North Carolina State University, Chapel Hill, North Carolina, \\ United States \\ Corresponding Author: Jason R. Franz \\ Email address: jfranz@email.unc.edu
}

The human Achilles tendon (AT) consists of sub-tendons arising from the gastrocnemius and soleus muscles that exhibit non-uniform tissue displacements thought to facilitate some independent actuation. However, the mechanisms governing non-uniform displacement patterns within the AT, and their relevance to triceps surae muscle contractile dynamics, have remained elusive. We used a dual-probe ultrasound imaging approach to investigate triceps surae muscle dynamics (i.e., medial gastrocnemius-GAS, soleus-SOL) as a determinant of non-uniform tendon tissue displacements in the human AT. We hypothesized that superficial versus deep differences in AT tissue displacements would be accompanied by and correlate with anatomically consistent differences in GAS versus SOL muscle shortening. Nine subjects performed ramped isometric contractions at each of five ankle joint angles spanning $10^{\circ}$ dorsiflexion to $30^{\circ}$ plantarflexion. For all conditions, SOL shortened by an average of $78 \%$ more than GAS during moment generation. This was accompanied by, on average, 51\% more displacement in the deep versus superficial region of the AT. The magnitude of GAS and SOL muscle shortening positively correlated with displacement in their associated sub-tendons within the AT. Moreover, and as hypothesized, superficial versus deep differences in sub-tendon tissue displacements positively correlated with anatomically consistent differences in GAS versus SOL muscle shortening. We present the first in vivo evidence that triceps surae muscle dynamics may precipitate non-uniform displacement patterns in the architecturally complex AT. 
1 Do Triceps Surae Muscle Dynamics Govern Non-uniform Achilles Tendon Deformations?

2

3 William H. Clark and Jason R. Franz

4

5 Joint Department of Biomedical Engineering, University of North Carolina at Chapel Hill and

6 North Carolina State University, Chapel Hill, NC, USA

7

8 Corresponding Author:

9 Jason R. Franz

10 Address: 152 MacNider Hall, CB 7575

11 Chapel Hill, NC 27599

12 Telephone: (919)966-6119

13 Fax: (919)966-2963

14 Email: jrfranz@email.unc.edu

17

18

19

20

21

22

23

24

25

26

27

28

29

30

31

Word count: 4166 


\section{ABSTRACT}

The human Achilles tendon (AT) consists of sub-tendons arising from the gastrocnemius

34 and soleus muscles that exhibit non-uniform tissue displacements thought to facilitate some

35 independent actuation. However, the mechanisms governing non-uniform displacement patterns

36 within the AT, and their relevance to triceps surae muscle contractile dynamics, have remained

37 elusive. We used a dual-probe ultrasound imaging approach to investigate triceps surae muscle

38 dynamics (i.e., medial gastrocnemius-GAS, soleus-SOL) as a determinant of non-uniform tendon

39 tissue displacements in the human AT. We hypothesized that superficial versus deep differences

40 in AT tissue displacements would be accompanied by and correlate with anatomically consistent

41 differences in GAS versus SOL muscle shortening. Nine subjects performed ramped isometric

42 contractions at each of five ankle joint angles spanning $10^{\circ}$ dorsiflexion to $30^{\circ}$ plantarflexion. For

43 all conditions, SOL shortened by an average of 78\% more than GAS during moment generation.

44 This was accompanied by, on average, 51\% more displacement in the deep versus superficial 45 region of the AT. The magnitude of GAS and SOL muscle shortening positively correlated with 46 displacement in their associated sub-tendons within the AT. Moreover, and as hypothesized, 47 superficial versus deep differences in sub-tendon tissue displacements positively correlated with 48 anatomically consistent differences in GAS versus SOL muscle shortening. We present the first in 49 vivo evidence that triceps surae muscle dynamics may precipitate non-uniform displacement 50 patterns in the architecturally complex AT.

51

52 
55

56

57

58

59

60

61

62

63

64

65

66

67

68

69

70

71

72

73

74

75

76

77

\section{INTRODUCTION}

The Achilles tendon (AT) is a critical passive elastic structure that transmits contractile forces from the gastrocnemius and soleus muscles (i.e., triceps surae) to generate a moment about the ankle, thereby powering activities such as walking (Fukashiro et al., 2006). In young adults, the power generated via triceps surae muscle-tendon interaction during walking is responsible for $70 \%-80 \%$ of the mechanical power needed for forward propulsion and swing initiation (Neptune et al., 2009; Whittington et al., 2008; Zelik et al., 2014). The architecturally complex AT consists of three distinct bundles of tendon fascicles, known as "sub-tendons" (Handsfield et al., 2017), arising from the medial and lateral gastrocnemius and soleus muscles (Bojsen-Moller and Magnusson, 2015; Edama et al., 2015; Szaro et al., 2009). Recent advances in the use of dynamic, in vivo ultrasound imaging have revealed non-uniform displacement patterns within the AT. Those non-uniform patterns are commonly interpreted as evidence for sliding between adjacent subtendons, which has the potential to facilitate independence between the individual triceps surae muscles (Arndt et al., 2012; Chernak Slane and Thelen, 2014; Franz and Thelen, 2015). However, the mechanisms governing these non-uniform displacement patterns, and their relevance to triceps surae muscle contractile dynamics, remain elusive.

To our knowledge, no empirical studies have characterized the origins of sliding between adjacent sub-tendons within the human AT. However, recent model predictions implicate differential gastrocnemius versus soleus muscle dynamics as a plausible candidate (Handsfield et al., 2017). Using anatomy derived from MRI, Handsfield et al. (2017) tuned a finite-element model of the AT to replicate published estimates of in vivo AT tissue displacements during isolated contractions (Chernak Slane and Thelen, 2014). The authors interrogated their model to reveal that without differential muscle forces, AT tissue non-uniformity decreased by $85 \%$ (Handsfield et al., 
78 2017). One implication of this prediction is that triceps surae muscle dynamics may precipitate

79 non-uniform displacement patterns in the human AT - a finding for which experimental evidence

80 has not yet been presented. Indeed, other factors, including differences in sub-tendon material or

81 architectural properties or in calcaneal insertion have also been implicated (Franz and Thelen,

82 2015; Thorpe et al., 2012, 2013).

83 Cine B-mode imaging has revealed important disparities between the individual triceps

84 surae muscles. For example, Ishikawa et al. (2005) found that gastrocnemius muscle fascicles

85 remain isometric or shorten during the late stance phase of walking while soleus muscle fascicles

86 lengthen (Ishikawa et al., 2005b). Cronin et al. (2013) added that the contractile behavior of the

87 gastrocnemius, but not the soleus, changed with walking speed (Cronin et al., 2013). These data

88 allude to the potential for functional benefits at the muscle level conferred by independent 89 actuation - actuation that may be facilitated by the AT itself (Huijing et al., 2011; Kawakami et 90 al., 1998; Tian et al., 2012). However, we recently observed cine B-mode imaging of 91 gastrocnemius and soleus muscle dynamics alone are unable to reliably estimate interactions

92 between these muscles and their series elastic sub-tendons (Zelik and Franz, 2017). Thus, to gain 93 mechanistic insight into the role of triceps surae muscle dynamics in precipitating non-uniform 94 displacement patterns within the AT, there is a critical need for innovation in our measurement 95 techniques; to understand the complexities of muscle and tendon behavior, you must measure 96 muscle and tendon behavior.

97 Our goal was to investigate individual triceps surae (i.e., medial gastrocnemius and soleus) 98 muscle dynamics as a determinant of non-uniform AT tissue displacement patterns during isolated 99 contractions. We used a dual-probe ultrasound imaging technique to quantify in vivo 100 gastrocnemius and soleus muscle dynamics in synchrony with localized tendon tissue 
101 displacements within their associated sub-tendons. We hypothesized that superficial versus deep

102 differences in AT tissue displacements would be accompanied by and correlate with anatomically

103 consistent differences in medial gastrocnemius (GAS) versus soleus (SOL) muscle shortening. We

104 also tested the secondary hypothesis that the relationship between AT tissue displacements and

105 GAS versus SOL muscle shortening would vary with ankle angle, which we would interpret in the

106 context of altered triceps surae force generating capacity (Huijing et al., 2011; Kawakami et al.,

107 1998; Tian et al., 2012) and tendon slack (Herbert et al., 2011; Hug et al., 2013).

108 2. MATERIALS AND METHODS

109 2.1. Subjects and protocol

110 We estimated that $\mathrm{n}=8$ subjects would have $80 \%$ power to detect $(\mathrm{p}<0.05)$ previously

111 reported differences between peak GAS and SOL sub-tendon displacements (i.e., $5.95 \mathrm{~mm}$ versus

$1128.49 \mathrm{~mm}$ ) in young subjects during an isolated ankle task (Slane and Thelen, 2015). We recruited

11312 subjects to participate and excluded 3 subjects during our quality control process. Specifically,

114 we excluded subjects for whom the average frame-to-frame correlation in our speckle tracking

115 algorithm, later described, for any one condition fell below $80 \%$. Thus, we report data for 9

116 subjects (age: $25.1 \pm 5.6$ years, mass: $69.8 \pm 6.9 \mathrm{~kg}$, height: $1.7 \pm 0.1 \mathrm{~m}, 4$ females and 5 males).

117 Subjects provided written informed consent as per the UNC Biomedical Sciences Institutional

118 Review Board (16-0379) and walked without an assistive aid, had no orthopedic disorders within

119 the last six months nor any neurological disorder or disease, and did not have a leg prosthesis.

120 Subjects first walked on a treadmill (Bertec Corp., Columbus, OH) for $6 \mathrm{~min}$ at $1.25 \mathrm{~m} / \mathrm{s}$ to pre-

121 condition their triceps surae muscle-tendon units and reach steady-state behavior (Hawkins et al.,

122 2009). Subjects then performed three ramped maximum isometric voluntary contractions at each

123 of five ankle angles $\left(10^{\circ}\right.$ dorsiflexion to $30^{\circ}$ plantarflexion in $10^{\circ}$ increments) in a dynamometer 
124 (Biodex, Shirly, NY), with the knee flexed to replicate that near the push-off phase of walking

125 ( $\sim 20$ degrees). To elicit a symmetric loading-unloading profile, subjects started from rest and

126 increased their plantarflexor moment until reaching their voluntary maximum at 2 seconds, before

127 steadily returning to rest at 4 seconds. Prior to data collection, subjects briefly practiced this 4

128 second ramped contraction using a real-time display of their net ankle moment for positive

129 reinforcement. We fully-randomized the ankle angles, and subjects rested at least one minute

130 between each contraction. Subjects were barefoot throughout the experiment to allow proper

131 placement of the ultrasound transducers.

132 2.2. Measurements

133 We synchronized two $10 \mathrm{MHz}$ linear array ultrasound transducers to simultaneously record

134 GAS and SOL fascicle kinematics with tissue displacements in their associated tendinous

135 structures (Fig. 1). A 60 mm Telemed Echo Blaster 128 ultrasound transducer (LV7.5/60/128Z-2,

136 UAB Telemed, Vilnius, Lithuania) placed over the mid-belly of the GAS of subjects' right leg

137 recorded cine B-mode images at 61 frames/s through a longitudinal cross section using an image

138 depth of $65 \mathrm{~mm}$. This Telemed transducer placement and depth also enabled imaging of the SOL

139 in the same image plane (Cronin et al., 2013; Tian et al., 2012). Simultaneously, a 38-mm

140 transducer (L14-5W/38, Ultrasonix Corporation, Richmond, BC) operating at 70 frames/s

141 recorded 128 lines of ultrasound radiofrequency (RF) data from subjects' right free AT, distal to

142 the SOL muscle-tendon junction, using an image depth of $20 \mathrm{~mm}$ and secured using a custom

143 orthotic. Placement of this probe was prescribed by the fit of our custom orthotic, secured with

144 straps just proximal to the malleoli and thus selected to replicate the placement used in prior studies

145 (i.e., $\sim 6 \mathrm{~cm}$ proximal to the calcaneal insertion) (Franz et al., 2015). 
147 Rosa, CA) operating at $100 \mathrm{~Hz}$ recorded the 3D positions of 14 retroreflective markers placed on

148 the subjects' right lower leg and ultrasound transducers. An inverse kinematics routine (Silder et

149 al., 2008) estimated ankle and knee joint angles and we recorded the plantarflexor moment from 150 the dynamometer at $1000 \mathrm{~Hz}$.

151 We collected binary synchronization signals from the Telemed and Ultrasonix machines at $1521000 \mathrm{~Hz}$. These signals co-registered the onset of ultrasound data collected from the GAS, SOL, 153 and free AT with the plantarflexor moment and marker trajectories. We analyzed all data between 154 key-frames at the beginning and end of each ramped contraction, defined using a threshold of 5\% 155 peak moment. From the ultrasound data, we quantified time series of (i) GAS and SOL fascicle 156 kinematics and (ii) tendon tissue kinematics, each interpolated to 1000 data points per trial, as 157 described below.

158

159

160

161

162

163

164 165 166 167

\subsection{Muscle kinematics}

Following best practices outlined by Farris and Lichtwark (2016), the same investigator performed all muscle tracking. First, we defined a static polygon region of interest (ROI) surrounding each muscle and their aponeuroses (Fig. 1). We then defined one GAS and one SOL muscle fascicle in the mid-region of the imaged plane, considered representative of the muscle belly, from their superficial to deep aponeurosis in the first "key-frame" of every trial. Open source MATLAB routines based on an affine extension to an optic flow algorithm quantified time series of GAS and SOL fascicle lengths $\left(L_{\text {fascicle }}\right)$ and pennation angles $(\alpha)$ (Farris and Lichtwark, 2016). Pennation angles corresponded to the oblique angle between the image horizontal axis and the defined fascicle of the respective muscle. Finally, to more directly place these muscle dynamics in 
168 the context of tendon tissue displacement, we combined muscle fascicle lengths and pennation

169 angles to compute muscle length longitudinal to its line of action $\left(L_{\text {muscle }}\right)$ :

$170 \quad L_{\text {muscle }}=L_{\text {fascicle }} \cos \alpha$

\section{2.4. Tendon kinematics}

172

A 2D speckle tracking algorithm estimated localized displacements of AT tissue using

173 previously published techniques (Chernak and Thelen, 2012; Chernak Slane and Thelen, 2014). In

174 brief, we defined a rectangular ROI, measuring $\sim 15 \times 3 \mathrm{~mm}$ on a B-mode image of the free AT

175 created from the raw RF data at the first "key-frame" of each trial. The ROI contained a grid of

176 nodes with $0.83 \times 0.42 \mathrm{~mm}$ spacing defined to encompass only tendinous tissue. A $2 \mathrm{~mm} \times 1 \mathrm{~mm}$

177 kernel containing up-sampled (4x) RF data, centered at each nodal position, provided a search

178 window over which we defined two-dimensional (2D) normalized cross-correlation functions

179 between successive frames. We defined localized frame-to-frame nodal displacements that

180 maximized these 2D cross-correlations, with the cumulative displacement representing the average

181 of forward and backward tracking results. Nodal displacements were regularized using second

182 order polynomials (Pan et al., 2009). From these cumulative displacements, we quantified the

183 average longitudinal nodal displacements originating from two equally sized tendon depths (Szaro

184 et al., 2009) - superficial and deep - corresponding to tendon tissue thought to arise from GAS and

185 SOL, respectively. This orientation represents the anatomical arrangement most prevalent in

186 cadaveric studies (Anson and McVay, 1971; Edama et al., 2015; Gils et al., 1996; Szaro et al.,

187 2009). Although previous authors have acknowledged that in some anatomical observations the

188 individual sub-tendons varied in their thickness, in the majority of the anatomical observations,

189 the gastrocnemius sub-tendon was the same size as the soleus sub-tendon (Anson and McVay,

190 1971; Del Buono et al., 2013; Doral et al., 2010; Gils et al., 1996). Here, we report these average 
191 longitudinal displacements as representing that of tissue within the corresponding GAS and SOL

192 sub-tendons (Fig. 1). Finally, we quantified tendon non-uniformity by reporting the difference

193 between peak tendon tissue displacements of the superficial and deep regions of the tendon.

194 2.5. Statistical analysis

195 For each outcome measure, we took the average of the three conditions for each ankle 196 angle. A two-way repeated measures ANOVA tested for main effects of and interactions between 197 ankle angle and muscle-tendon unit (i.e., GAS and SOL) on peak muscle shortening and peak 198 tendon tissue displacement using an alpha level of 0.05 . When a significant main effect was found, 199 post-hoc pairwise comparison identified the ankle angles at which GAS versus SOL differences 200 were significant. For peak plantarflexor moment, a one-way repeated measures ANOVA tested for 201 significant main effects of ankle angle and Pearson's correlation coefficients assessed the relation 202 to muscle and sub-tendon tissue kinematics. We then calculated Pearson's correlation coefficients 203 between: (i) GAS and SOL muscle shortening and displacement in their associated regions of the 204 AT (i.e., superficial and deep, respectively) and (ii) muscle shortening differences (i.e., GAS 205 versus SOL) and tendon non-uniformity (i.e., superficial versus deep). As a secondary analysis, 206 we calculated Pearson's correlation coefficients between GAS and SOL muscle shortening and 207 displacement in their unassociated region of the AT (i.e., GAS versus deep, SOL versus 208 superficial). Finally, we qualitatively assessed the independent contributions of fascicle length 209 versus pennation angle on GAS and SOL muscle length changes. We plotted percent change in 210 fascicle length versus percent change in the cosine of pennation angle (i.e., contributors to muscle 211 length change) and noted apparent trends against the line of unity with a ratio of 1:1.

\section{3. RESULTS}



across the range of angles tested $(p<0.01)$. Muscle and sub-tendon tissue kinematics were relatively 215 independent of ankle angle; only SOL fascicle and longitudinal muscle shortening exhibited 216 significant main effects $\left(p^{\prime} s^{\prime}=0.03\right)$, with pairwise comparisons revealing smaller changes at $30^{\circ}$ 217 plantarflexion compared to the other conditions. However, consistent with this observation, SOL 218 tendon displacements also tended to be smaller at $30^{\circ}$ plantarflexion $(p=0.064)$. Across all ankle 219 angles, the SOL shortened by an average of 78\% more than the GAS during moment generation $220(p<0.01)$ (Fig. 2A), due both to greater fascicle shortening and a larger increase in pennation $221(p ’ s<0.01)$ (Fig. 3). However, change in fascicle length had a greater influence than change in the 222 cosine of pennation on longitudinal GAS and SOL muscle shortening (Fig. 3).

224 more displacement in the deep versus superficial region of the AT $(p<0.01)$ (Fig. 2B). Moreover, 225 the magnitude of peak GAS and SOL muscle shortening positively correlated with peak 226 displacements in their associated regions of the AT (GAS: $\mathrm{R}^{2}=0.48 ; \mathrm{SOL}: \mathrm{R}^{2}=0.63 ; p$ ' $\left.<0.01\right)$ (Fig. 227 4A). Superficial versus deep differences in AT displacement positively correlated with 228 anatomically consistent differences in GAS versus SOL muscle shortening $\left(\mathrm{R}^{2}=0.37, p<0.01\right)$ (Fig. 229 4B). The strength of these latter correlations varied systematically with ankle angle, becoming 230 stronger with increased ankle dorsiflexion (Table 1). Conversely, those correlations became non231 significant at $20^{\circ}(p=0.10)$ and $30^{\circ}(p=0.99)$ plantarflexion. We also found moderate to strong 232 correlations between GAS and SOL muscle shortening and displacement in their unassociated 233 region of the AT $\left(\mathrm{R}^{2}=0.38\right.$ and $\mathrm{R}^{2}=0.65$, respectively; $p$ 's $\left.<0.05\right)$. Finally, across all conditions, 234 peak muscle shortening $\left(\mathrm{GAS}: \mathrm{R}^{2}=0.37\right.$; $\left.\mathrm{SOL}: \mathrm{R}^{2}=0.47\right)$ and sub-tendon tissue displacements 
235 (GAS: $\mathrm{R}^{2}=0.51$; SOL: $\left.\mathrm{R}^{2}=0.49\right)$ significantly and positively correlated with peak plantarflexor 236 moment.

\section{4. DISCUSSION}

238 We investigated the role of triceps surae muscle dynamics in precipitating non-uniform 239 tissue displacements in the architecturally complex Achilles tendon (AT). We also used a dual240 probe ultrasound imaging technique empowering the simultaneous measurement of GAS and SOL 241 muscle fascicle dynamics together with tissue displacements within their associated sub-tendons 242 of the AT. Our results build on dual-probe approaches applied previously to GAS quantifying 243 muscle fascicle and muscle-tendon junction kinematics (Ishikawa and Komi, 2008; Matijevich et 244 al., 2018). Our findings largely supported our first hypothesis; independent of ankle angle, SOL 245 shortened more than GAS during moment generation - muscle-level differences were 246 accompanied by and correlated with anatomically consistent differences in sub-tendon tissue 247 displacements. As we elaborate, these findings provide empirical evidence for a mechanistic link 248 between non-uniform displacement patterns within the human AT and the operating behavior of 249 the triceps surae muscles.

250 Previous anatomical work has shown that the human free AT near our imaging location 251 consists predominantly of superficial tendon fascicles arising from GAS and deep tendon fascicles 252 arising from SOL (Szaro et al., 2009). Consistent with this architecture, the SOL muscle and its 253 associated deep region of the AT in all cases both underwent larger kinematic changes during 254 moment generation compared to those structures associated with GAS. Although not previously 255 quantified simultaneously, these triceps surae muscle (Fukashiro et al., 2006; Hoang et al., 2007; 256 Lauber et al., 2014; Maganaris et al., 1998) and AT tissue (Franz et al., 2015) kinematics are 257 generally consistent with those reported for a variety of activities spanning isolated contractions 
258 to functional activities. For example, we reported larger tissue displacements in the deep AT (i.e., 259 SOL sub-tendon) than in the superficial AT (i.e., GAS sub-tendon) during the stance phase of 260 walking, a phase in which GAS and SOL muscle fascicles exhibit different operating behavior 261 (Ishikawa et al., 2005a). In their review, Bojsen-Møller and Magnusson (2015) summarize 262 evidence for heterogeneous loading within the AT and at least conceptualize the potential for 263 differential GAS and SOL activations, and thus muscle contractile dynamics, to precipitate non264 uniform patterns of AT tissue displacements (Bojsen-Moller and Magnusson, 2015).

265 Despite conceptual descriptions (Bojsen-Moller and Magnusson, 2015) and recent model 266 predictions (Handsfield et al., 2017), we lacked evidence for the role of muscle dynamics in 267 governing non-uniform mechanical behavior within the AT. Consistent with our first hypothesis, 268 here we found that differences between peak SOL and GAS muscle shortening positively 269 correlated with those between peak SOL and GAS sub-tendon tissue displacement during fixed270 end contractions. Coupled with anatomically consistent differences in muscle shortening and 271 tendon tissue displacements described above, these correlations suggest that triceps surae muscle 272 dynamics can give rise to sliding between adjacent sub-tendons within the human AT.

273 We qualitatively assessed the contributions of fascicle length versus pennation angle to 274 differences between GAS and SOL muscle contractile behavior. Fascicle length and pennation 275 angles of the GAS and SOL, including their changes during isometric contractions, agree well with 276 published values (Maganaris, 2003; Maganaris et al., 1998; Tilp et al., 2011). In all cases, fascicle 277 shortening contributed more than fascicle rotation to the longitudinal GAS and SOL muscle length 278 changes. Moreover, the SOL exhibited both larger fascicle shortening and fascicle rotation than 279 GAS. In fact, we noted very little pennation angle change in the GAS during moment generation. 280 Thus, differences in triceps surae contractile behavior arise from a combination of larger fascicle 
281 shortening and rotation in SOL versus GAS, contributing to the non-uniform length change 282 dynamics ultimately borne by the AT. versus SOL muscle differences in shortening increased with ankle dorsiflexion, which we interpret as support for our secondary hypothesis. Correlations were strongest at the most dorsiflexed ankle angle (i.e., $10^{\circ}$ ), decreasing progressively with increasing plantarflexion. This observation may reflect the effects of decreased tendon slack, decreased muscle passive tension, and/or larger peak moments conveyed by increasing ankle dorsiflexion. Indeed, muscle and tendon mechanical behavior may exhibit complex changes in response to ankle rotation (Hug et al., 2013). More tendon slack would imply greater tendon length change, and thus tendon tissue displacements, prior to the onset of force transmission (Zajac, 1989). We suspect that differences in tendon slack explain the relatively invariant tendon kinematics across the range of ankle angles tested, despite systematic changes in peak moment. Similarly, more tendon slack and/or differences between GAS and SOL tendon slack lengths may explain why these correlations became insignificant with increasing ankle plantarflexion. We also found that ankle dorsiflexion maximized peak moment, and presumably AT force transmission, consistent with prior studies (Ackland et al., 2012; Scovil and Ronsky, 2006; Zajac, 1989). Thus, larger forces may themselves convey stronger correlations between muscle and tendon tissue dynamics. Simultaneously, or perhaps alternatively, ankle angle effects on the mechanical properties of the individual triceps surae muscles and sub-tendons themselves could influence the relation between muscle and tendon behavior. Additional study here is certainly warranted. 
304 connective tissues between these muscles is also prevalent. Oda et al. (2007) found that an isolated

305 stimulus to GAS elicited a similar time course and magnitude of length change in both GAS and

306 SOL (Oda et al., 2007). Kinugasa et al. (2013) also suggested that interaponeurosis shear, elicited

307 by adjacent muscle dynamics, influences displacements of the distal muscle-tendon junctions

308 (Kinugasa et al., 2013). More recently, Finni et al. (2017) added that the strength of this lateral

309 connectivity increases with muscle activation (Finni et al., 2017). However, non-uniform AT

310 displacement patterns are also present during passive ankle rotation in the absence of triceps surae

311 muscle loading (Arndt et al., 2012; Chernak Slane and Thelen, 2014). For this study, we interpret

312 our findings in the context of their relevance to independent actuation; follow-up studies are

313 necessary to better understand the relative role of lateral force transmission. Indeed, we found that

314 SOL muscle shortening exhibited strong positive correlations with GAS sub-tendon displacement,

315 and vice versa. One interpretation of this finding is that SOL, having by far the largest force-

316 generating capacity of the triceps surae muscles (Albracht et al., 2008; Ogihara et al., 2017)

317 coupled with having greater fascicle shortening and rotation than GAS, has a preferential influence

318 on tendon tissue displacements in the free AT. Taking together, our results reflect independent and

319 inter-dependent mechanical behavior between individual triceps surae muscle-tendon units.

320 Indeed, intermuscular force transmission is documented within the triceps surae (Huijing et al.,

321 2011; Tian et al., 2012).

322 There are other possible factors contributing to AT tissue non-uniformity that may act in

323 concert with triceps surae muscle dynamics. Material property differences between adjacent sub-

324 tendons may elicit non-uniform displacement patterns. Indeed, Matson et al. (2012) reported up to

325 two-fold variations in elastic modulus between different leg tendons (Matson et al., 2012). In

326 addition, architectural differences between sub-tendons (e.g., lengths and cross-sectional areas) 
327 may influence their mechanical properties (e.g., stiffness) and thus displacement patterns (Szaro

328 et al., 2009). As another example, differences between sub-tendon slack lengths could also

329 contribute. We consider the present study a first step toward an improved understanding of the

330 relative contributions of these factors in governing triceps surae muscle-tendon behavior.

331 If triceps surae muscle dynamics can precipitate non-uniform Achilles tendon tissue

332 displacements, can tendon-level changes influence muscle contractile behavior? For example, our

333 findings may be important for understanding the functional consequences of age-associated

334 reductions in inter-fascicle sliding within the human AT. Compared to those in young adults, we

335 have observed more uniform AT tissue displacements in older adults during walking (Franz and

336 Thelen, 2015), which may reflect collagen cross-linking and interfascicle adhesions (Thorpe et al.,

337 2013). These tendon-level changes correlate with a reduced plantarflexor moment during walking

338 in older adults, alluding to unfavorable functional consequences. Moreover, simulating a reduced

339 capacity for inter-fascicle sliding in the AT, and thus a loss of mechanical independence between

340 the GAS and SOL, predicts unfavorable shifts in muscle fascicle behavior during walking (Franz

341 and Thelen, 2016). Our present work provides a foundation for using dual-probe imaging to gain

342 mechanistic insight into these earlier observations.

343 There are several limitations of this study. First, we only report data for the medial

344 gastrocnemius and SOL, including a generalized anatomical approximation of their associated sub-

345 tendons (Edama et al., 2015; Gils et al., 1996; Szaro et al., 2009). Second, two-dimensional

346 imaging may not fully capture the three-dimensional behavior of the triceps surae muscles and AT.

347 For example, the SOL muscle is comprised of anterior, posterior, medial, and lateral components

348 that differ in architecture (Chow et al., 2000). We only imaged the posteromedial SOL, which may

349 not represent other regions. Third, muscle tracking reliability depends on meticulous fascicle 
350 determination and incumbent semi-automated tracking limitations (Farris and Lichtwark, 2016).

351 To minimize these effects, one investigator performed all analyses. Fourth, the pennation angle

352 defined by UltraTrack is not representative of a true pennation angle due to the software identifying

353 the line of action as the horizontal axis of the image. We have also previously described the

354 limitations of our 2D speckle tracking estimates of AT tissue displacements (Franz et al., 2015).

355 We add here that cross-correlation estimates of tissue motion can be subject to spatial averaging.

356 However, based on a previously published validation (Chernak and Thelen, 2012; Chernak Slane

357 and Thelen, 2014), we do not suspect this significantly influenced our findings nor interpretation.

358 We also opted to report sub-tendon tissue displacements, an outcome we can measure with a higher

359 level of confidence than sub-tendon elongation. Specifically, estimating sub-tendon tissue

360 elongation relative to a motion capture estimate of calcaneal insertion can be prone to errors

361 associated with coronal plane ankle rotation, tendon slack, and the complexities of AT curvature -

362 errors that require further study (Csapo et al., 2013; Matijevich et al., 2018). Fifth, we made no

363 attempt to estimate forces transmitted through the AT, which are heterogeneous and highly

364 complex (Bojsen-Moller and Magnusson, 2015). We also note that ankle and knee angles became

365 slightly $\left(<5^{\circ}\right)$ more extended from rest to peak moment generation. The sub-tendon tissue

366 displacements reported here likely reflect some combination of that due to muscle shortening at

367 the proximal attachment and that due to calcaneus displacement (via ankle rotation) at the distal

368 attachment. However, Handsfield et al. (2017) found a very negligible effect of retrocalcaneal

369 tendon insertion on GAS-SOL differences in sub-tendon tissue displacement, even for $25^{\circ}$ of ankle

370 rotation (Handsfield et al., 2017). Finally, although we report anatomically consistent behavior

371 between muscle and sub-tendons, our conclusions are based in part on correlations that cannot

372 definitively convey causal links. 


\section{5. CONCLUSIONS}

374 We present evidence that triceps surae muscle dynamics may precipitate non-uniform

375 displacement patterns in the architecturally complex Achilles tendon. Moreover, we used a dual-

376 probe imaging approach to empower simultaneous assessment of muscle and tendon toward an

377 improved mechanistic understanding of triceps surae behavior. Our findings may be important for

378 understanding age-associated changes in AT displacement patterns, which we suspect alter muscle

379 contractile behavior in older adults.

380

381

\section{ACKNOWLEDGEMENTS}

382

We thank Ashish Khanchandani, Hannah McKenney, and Michael Browne for their

383

assistance with data collection.

384

385

386

387

388

389

390

391

392

393

394

395 


\section{REFERENCES}

401

402

403

404

405

406

407

408

409

410

411

412

413

414

415

416

417

418

419

420

421

422

423

424

425

426

427

428

429

430

431

432

433

434

435

Ackland, D.C., Lin, Y.C., Pandy, M.G., 2012. Sensitivity of model predictions of muscle function to changes in moment arms and muscle-tendon properties: a Monte-Carlo analysis. J Biomech 45, 1463-1471.

Albracht, K., Arampatzis, A., Baltzopoulos, V., 2008. Assessment of muscle volume and physiological cross-sectional area of the human triceps surae muscle in vivo. J Biomech 41, 22112218.

Anson, B.J., McVay, C.B., 1971. Surgical Anatomy. Leg. (5th ed.). W.B. Saunders Company, Philadelphia, London, Toronto (1971), 1186-1189.

Arndt, A., Bengtsson, A.S., Peolsson, M., Thorstensson, A., Movin, T., 2012. Non-uniform displacement within the Achilles tendon during passive ankle joint motion. Knee Surg Sports Traumatol Arthrosc 20, 1868-1874.

Bojsen-Moller, J., Magnusson, S.P., 2015. Heterogeneous Loading of the Human Achilles Tendon In Vivo. Exerc Sport Sci Rev 43, 190-197.

Chernak, L.A., Thelen, D.G., 2012. Tendon motion and strain patterns evaluated with twodimensional ultrasound elastography. J Biomech 45, 2618-2623.

Chernak Slane, L., Thelen, D.G., 2014. The use of 2D ultrasound elastography for measuring tendon motion and strain. J Biomech 47, 750-754.

Cronin, N.J., Avela, J., Finni, T., Peltonen, J., 2013. Differences in contractile behaviour between the soleus and medial gastrocnemius muscles during human walking. J Exp Biol 216, 909-914.

Csapo, R., Hodgson, J., Kinugasa, R., Edgerton, V.R., Sinha, S., 2013. Ankle morphology amplifies calcaneus movement relative to triceps surae muscle shortening. J Appl Physiol (1985) $115,468-473$.

Del Buono, A., Chan, O., Maffulli, N., 2013. Achilles tendon: functional anatomy and novel emerging models of imaging classification. Int Orthop 37, 715-721.

Doral, M.N., Alam, M., Bozkurt, M., Turhan, E., Atay, O.A., Donmez, G., Maffulli, N., 2010. Functional anatomy of the Achilles tendon. Knee Surg Sports Traumatol Arthrosc 18, 638-643.

Edama, M., Kubo, M., Onishi, H., Takabayashi, T., Inai, T., Yokoyama, E., Hiroshi, W., Satoshi, N., Kageyama, I., 2015. The twisted structure of the human Achilles tendon. Scand J Med Sci Sports 25, e497-503.

Farris, D.J., Lichtwark, G.A., 2016. UltraTrack: Software for semi-automated tracking of muscle fascicles in sequences of B-mode ultrasound images. Comput Methods Programs Biomed 128, 111-118.

Finni, T., Cronin, N.J., Mayfield, D., Lichtwark, G.A., Cresswell, A.G., 2017. Effects of muscle activation on shear between human soleus and gastrocnemius muscles. Scand J Med Sci Sports $27,26-34$. 
436 Franz, J.R., Slane, L.C., Rasske, K., Thelen, D.G., 2015. Non-uniform in vivo deformations of the 437 human Achilles tendon during walking. Gait Posture 41, 192-197.

438 Franz, J.R., Thelen, D.G., 2015. Depth-dependent variations in Achilles tendon deformations with 439 age are associated with reduced plantarflexor performance during walking. J Appl Physiol (1985) $440119,242-249$.

441 Franz, J.R., Thelen, D.G., 2016. Imaging and simulation of Achilles tendon dynamics: 442 Implications for walking performance in the elderly. J Biomech 49, 1403-1410.

443 Fukashiro, S., Hay, D.C., Nagano, A., 2006. Biomechanical behavior of muscle-tendon complex

444 during dynamic human movements. J Appl Biomech 22, 131-147.

Gils, C.C., Steed, R.H., Page, J.C., 1996. Torsion of the human Achilles tendon. J Foot Ankle Surg 35, 41-48.

448

449

Handsfield, G.G., Inouye, J.M., Slane, L.C., Thelen, D.G., Miller, G.W., Blemker, S.S., 2017. A 3D model of the Achilles tendon to determine the mechanisms underlying nonuniform tendon displacements. J Biomech 51, 17-25.

450

Hawkins, D., Lum, C., Gaydos, D., Dunning, R., 2009. Dynamic creep and pre-conditioning of the Achilles tendon in-vivo. J Biomech 42, 2813-2817.

452

Herbert, R.D., Clarke, J., Kwah, L.K., Diong, J., Martin, J., Clarke, E.C., Bilston, L.E., Gandevia, S.C., 2011. In vivo passive mechanical behaviour of muscle fascicles and tendons in human gastrocnemius muscle-tendon units. J Physiol 589, 5257-5267.

Hoang, P.D., Herbert, R.D., Todd, G., Gorman, R.B., Gandevia, S.C., 2007. Passive mechanical properties of human gastrocnemius muscle tendon units, muscle fascicles and tendons in vivo. $\mathrm{J}$ Exp Biol 210, 4159-4168.

458 Hug, F., Lacourpaille, L., Maisetti, O., Nordez, A., 2013. Slack length of gastrocnemius medialis 459 and Achilles tendon occurs at different ankle angles. J Biomech 46, 2534-2538.

Huijing, P.A., Yaman, A., Ozturk, C., Yucesoy, C.A., 2011. Effects of knee joint angle on global and local strains within human triceps surae muscle: MRI analysis indicating in vivo myofascial force transmission between synergistic muscles. Surg Radiol Anat 33, 869-879.

Ishikawa, M., Komi, P.V., 2008. Muscle fascicle and tendon behavior during human locomotion revisited. Exerc Sport Sci Rev 36, 193-199.

Ishikawa, M., Komi, P.V., Grey, M.J., Lepola, V., Bruggemann, G.P., 2005a. Muscle-tendon interaction and elastic energy usage in human walking. J Appl Physiol (1985) 99, 603-608. Ishikawa, M., Niemela, E., Komi, P.V., 2005b. Interaction between fascicle and tendinous tissues in short-contact stretch-shortening cycle exercise with varying eccentric intensities. J Appl Physiol (1985) 99, 217-223.

Kawakami, Y., Ichinose, Y., Fukunaga, T., 1998. Architectural and functional features of human triceps surae muscles during contraction. J Appl Physiol (1985) 85, 398-404.

473 Kinugasa, R., Oda, T., Komatsu, T., Edgerton, V.R., Sinha, S., 2013. Interaponeurosis shear strain modulates behavior of myotendinous junction of the human triceps surae. Physiol Rep 1, e00147. Lauber, B., Lichtwark, G.A., Cresswell, A.G., 2014. Reciprocal activation of gastrocnemius and soleus motor units is associated with fascicle length change during knee flexion. Physiol Rep 2. Maganaris, C.N., 2003. Force-length characteristics of the in vivo human gastrocnemius muscle. Clin Anat 16, 215-223.

Maganaris, C.N., Baltzopoulos, V., Sargeant, A.J., 1998. In vivo measurements of the triceps surae complex architecture in man: implications for muscle function. J Physiol 512 ( Pt 2), 603-614.

481 Matijevich, E.S., Branscombe, L.M., Zelik, K.E., 2018. Ultrasound estimates of Achilles tendon exhibit unexpected shortening during ankle plantarflexion. J Biomech 72, 200-206. 
482 Matson, A., Konow, N., Miller, S., Konow, P.P., Roberts, T.J., 2012. Tendon material properties

483

484

485

486

487

488

489

490

491

492

493

494

495

496

497

498

499

500

501

502

503

504

505

506

507

508

509

510

511

512

513

514

515

516

517

518

519

520

521

522

523 vary and are interdependent among turkey hindlimb muscles. J Exp Biol 215, 3552-3558.

Neptune, R.R., Clark, D.J., Kautz, S.A., 2009. Modular control of human walking: a simulation study. J Biomech 42, 1282-1287.

Oda, T., Kanehisa, H., Chino, K., Kurihara, T., Nagayoshi, T., Fukunaga, T., Kawakami, Y., 2007. In vivo behavior of muscle fascicles and tendinous tissues of human gastrocnemius and soleus muscles during twitch contraction. J Electromyogr Kinesiol 17, 587-595.

Ogihara, N., Oishi, M., Kanai, R., Shimada, H., Kondo, T., Yoshino-Saito, K., Ushiba, J., Okano, H., 2017. Muscle architectural properties in the common marmoset (Callithrix jacchus). Primates $58,461-472$.

Scovil, C.Y., Ronsky, J.L., 2006. Sensitivity of a Hill-based muscle model to perturbations in model parameters. J Biomech 39, 2055-2063.

Slane, L.C., Thelen, D.G., 2015. Achilles tendon displacement patterns during passive stretch and eccentric loading are altered in middle-aged adults. Med Eng Phys 37, 712-716.

Szaro, P., Witkowski, G., Smigielski, R., Krajewski, P., Ciszek, B., 2009. Fascicles of the adult human Achilles tendon - an anatomical study. Ann Anat 191, 586-593.

Thorpe, C.T., Udeze, C.P., Birch, H.L., Clegg, P.D., Screen, H.R., 2012. Specialization of tendon mechanical properties results from interfascicular differences. J R Soc Interface 9, 3108-3117.

Thorpe, C.T., Udeze, C.P., Birch, H.L., Clegg, P.D., Screen, H.R., 2013. Capacity for sliding between tendon fascicles decreases with ageing in injury prone equine tendons: a possible mechanism for age-related tendinopathy? Eur Cell Mater 25, 48-60.

Tian, M., Herbert, R.D., Hoang, P., Gandevia, S.C., Bilston, L.E., 2012. Myofascial force transmission between the human soleus and gastrocnemius muscles during passive knee motion. $\mathrm{J}$ Appl Physiol (1985) 113, 517-523.

Tilp, M., Steib, S., Schappacher-Tilp, G., Herzog, W., 2011. Changes in fascicle lengths and pennation angles do not contribute to residual force enhancement/depression in voluntary contractions. J Appl Biomech 27, 64-73.

Whittington, B., Silder, A., Heiderscheit, B., Thelen, D.G., 2008. The contribution of passiveelastic mechanisms to lower extremity joint kinetics during human walking. Gait Posture 27, 628634.

Zajac, F.E., 1989. Muscle and tendon: properties, models, scaling, and application to biomechanics and motor control. Crit Rev Biomed Eng 17, 359-411.

Zelik, K.E., Franz, J.R., 2017. It's positive to be negative: Achilles tendon work loops during human locomotion. PLoS One 12, e0179976.

Zelik, K.E., Huang, T.W., Adamczyk, P.G., Kuo, A.D., 2014. The role of series ankle elasticity in bipedal walking. J Theor Biol 346, 75-85. 
524

525

526

\section{Figure Captions}

528

529 Figure 1: We used a dual-probe ultrasound imaging approach that enables the simultaneous

530 assessment of the medial gastrocnemius (GAS) and soleus (SOL) muscle fascicle kinematics with

531 tissue displacements in their associated sub-tendons of the free Achilles tendon (AT). (A) Time

532 series of fascicle lengths and pennation angles were derived from cine B-mode images. (B) A

533 custom 2D speckle tracking algorithm estimated localized displacements of two equally sized

534 tendon depths - superficial and deep - corresponding to tendon tissue thought to arise from GAS 535 and SOL, respectively.

536

537 Figure 2: Group mean (standard error) (A) muscle shortening and (B) sub-tendon displacements 538 (nodal displacement relative to initial position; proximal positive) across the range of ankle angles 539 tested. Gray shaded regions show the group mean net profile during each loading-unloading cycle. 540 Across all conditions, SOL shortened by an average of $78 \%$ more than GAS during force 541 generation. This was accompanied by, on average, 51\% more lengthening in the deep versus 542 superficial region of the free AT. Asterisks $\left(^{*}\right)$ indicate statistically significant $(p<0.05)$ differences 543 between GAS and SOL.

544

545 Figure 3: Group mean percent change in fascicle length versus percent change in the cosine of 546 pennation angle for the gastrocnemius (GAS) and soleus (SOL) across the range of ankle angles 
547 tested. The dashed line represents a line of unity (i.e., equal contributions from fascicle shortening

548 and fascicle rotation). SOL consistently exhibited more fascicle shortening and fascicle rotation

549 than GAS. For all conditions, fascicle shortening had a greater influence on longitudinal muscle

550 shortening than fascicle rotation.

551

552 Figure 4: (A) Correlations between peak GAS or SOL muscle shortening and displacement in 553 their associated regions of the AT. (B) Correlation between superficial versus deep differences in 554 AT displacement and GAS versus SOL differences in muscle. All correlations are pooled across 555 all conditions.

556

557 


\section{Figure 1 (on next page)}

Dual-probe imaging methodological approach.

We used a dual-probe ultrasound imaging approach that enables the simultaneous assessment of the medial gastrocnemius (GAS) and soleus (SOL) muscle fascicle kinematics with tissue displacements in their associated sub-tendons of the free Achilles tendon (AT). (A) Time series of fascicle lengths and pennation angles were derived from cine B-mode images. (B) A custom 2D speckle tracking algorithm estimated localized displacements of two equally sized tendon depths - superficial and deep - corresponding to tendon tissue thought to arise from GAS and SOL, respectively. 


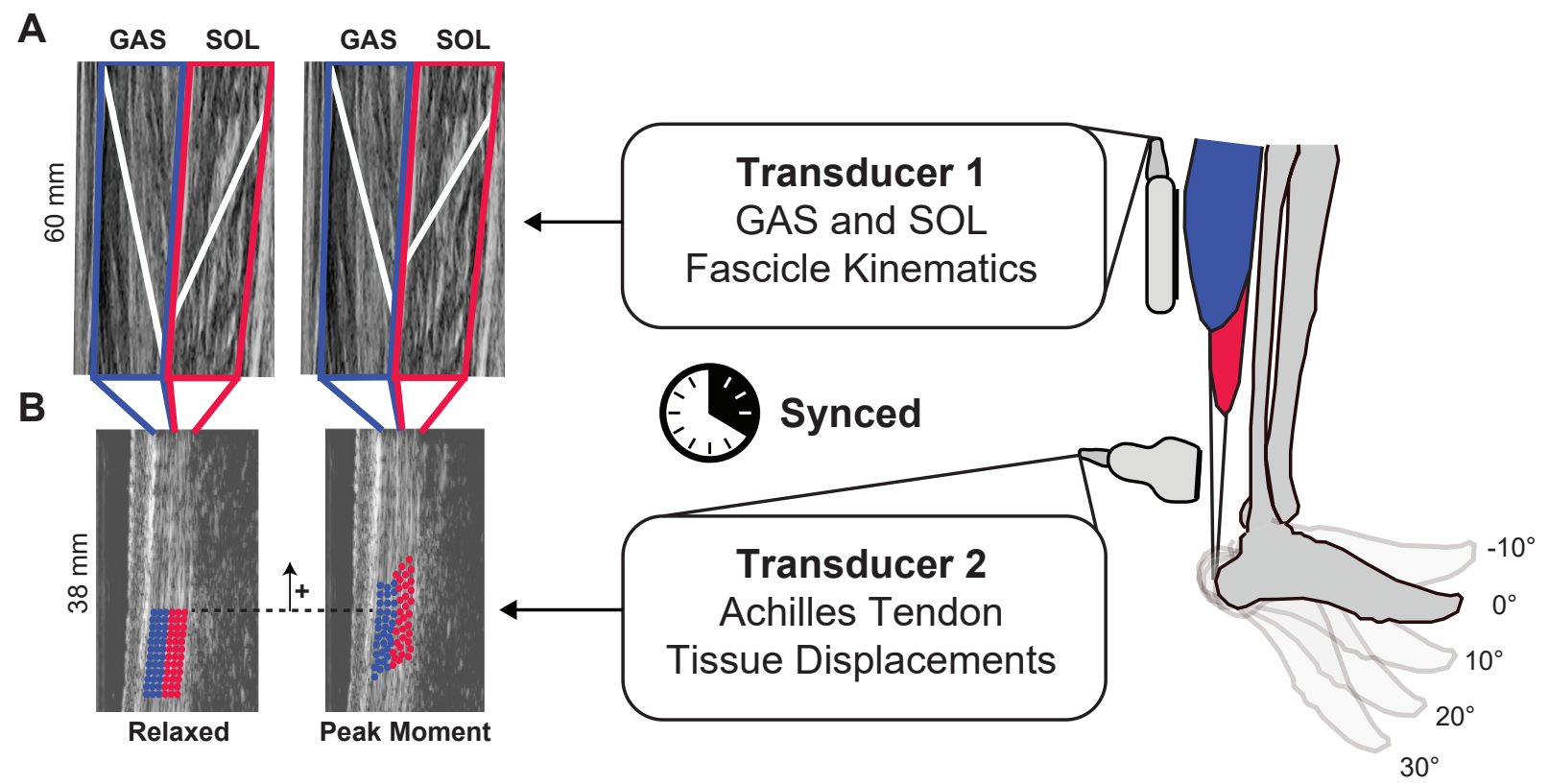




\section{Figure 2 (on next page)}

Muscle shortening and sub-tendon tissue displacements across the range of ankle angles tested.

Group mean (standard error) (A) muscle shortening and (B) sub-tendon displacements (nodal displacement relative to initial position; proximal positive) across the range of ankle angles tested. Gray shaded regions show the group mean net profile during each loading-unloading cycle. Across all conditions, SOL shortened by an average of $78 \%$ more than GAS during force generation. This was accompanied by, on average, 51\% more lengthening in the deep versus superficial region of the free AT. Asterisks $(*)$ indicate statistically significant $(p<0.05)$ differences between GAS and SOL. 


\section{Figure 3 (on next page)}

Contributions of fascicle length and pennation angle to muscle shortening behavior.

Group mean percent change in fascicle length versus percent change in the cosine of pennation angle for the gastrocnemius (GAS) and soleus ( $\mathrm{SOL}$ ) across the range of ankle angles tested. The dashed line represents a line of unity (i.e., equal contributions from fascicle shortening and fascicle rotation). SOL consistently exhibited more fascicle shortening and fascicle rotation than GAS. For all conditions, fascicle shortening had a greater influence on longitudinal muscle shortening than fascicle rotation. 

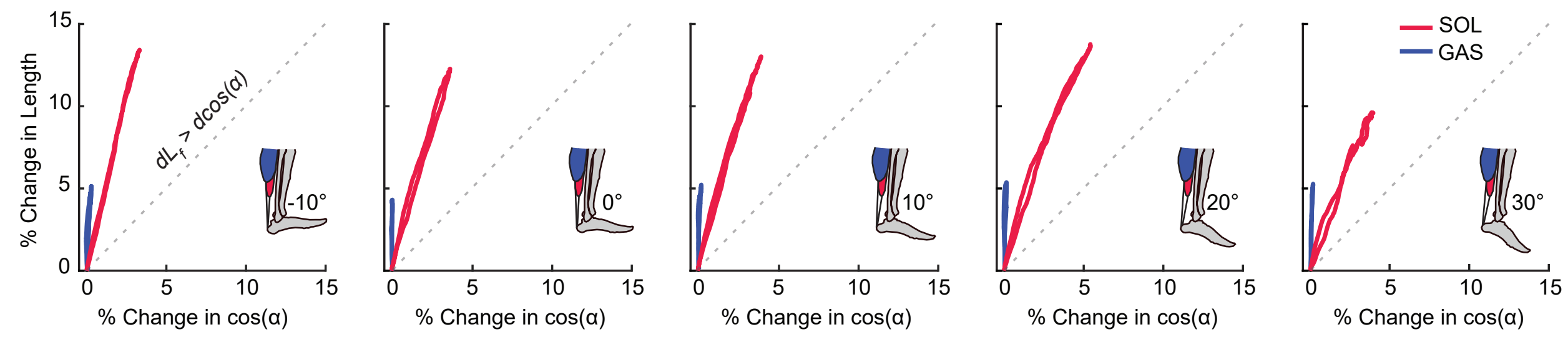
Figure 4 (on next page)

Correlations between muscle and subtendon tissue behavior.

(A) Correlations between peak GAS or SOL muscle shortening and displacement in their associated regions of the AT. (B) Correlation between superficial versus deep differences in AT displacement and GAS versus SOL differences in muscle. All correlations are pooled across all conditions. 


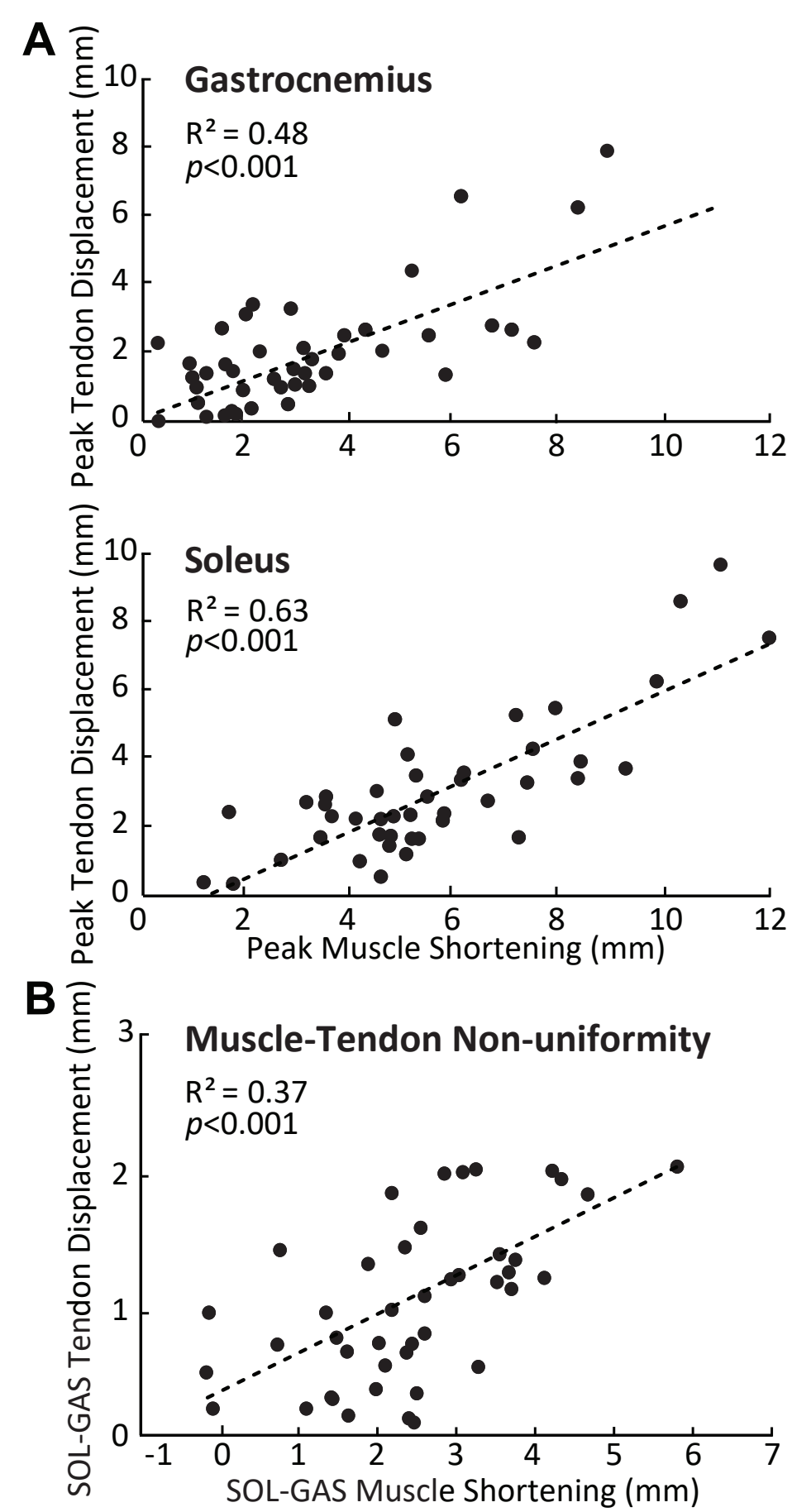




\section{Table $\mathbf{1}$ (on next page)}

Correlations between peak muscle shortening and peak sub-tendon tissue displacements at each ankle angle.

Bold numbers represent significant correlations $(p<0.05)$. GAS: Gastrocnemius, SOL: Soleus. 
Table 1. Correlations $\left(\mathrm{R}^{2}\right)$ and $p$-values between peak muscle shortening and peak sub-tendon tissue displacements at each ankle angle.

\begin{tabular}{clccccc}
\hline & & $-10^{\circ}$ & $0^{\circ}$ & $10^{\circ}$ & $20^{\circ}$ & $30^{\circ}$ \\
\hline \multirow{2}{*}{ GAS } & $\mathrm{R}^{2}$ & $\mathbf{0 . 5 2}$ & 0.20 & $\mathbf{0 . 6 7}$ & $\mathbf{0 . 5 0}$ & 0.32 \\
& $p$-value & $\mathbf{0 . 0 4 4}$ & 0.073 & $\mathbf{0 . 0 0 7}$ & $\mathbf{0 . 0 3 1}$ & 0.114 \\
\multirow{2}{*}{ SOL } & $\mathrm{R}^{2}$ & $\mathbf{0 . 7 7}$ & 0.38 & $\mathbf{0 . 7 5}$ & $\mathbf{0 . 7 6}$ & 0.36 \\
& $p$-value & $\mathbf{0 . 0 0 4}$ & 0.075 & $\mathbf{0 . 0 0 3}$ & $\mathbf{0 . 0 0 2}$ & 0.088 \\
\multirow{2}{*}{ SOL-GAS } & $\mathrm{R}^{2}$ & $\mathbf{0 . 7 8}$ & $\mathbf{0 . 6 0}$ & $\mathbf{0 . 4 5}$ & 0.33 & 0.04 \\
& $p$-value & $\mathbf{0 . 0 0 4}$ & $\mathbf{0 . 0 1 5}$ & $\mathbf{0 . 0 4 9}$ & 0.104 & 0.991 \\
\hline
\end{tabular}

Bold numbers represent significant correlations $(p<0.05)$.

GAS: Gastrocnemius, SOL: Soleus

1 